\section{International Scientific Journal Theoretical \& Applied Science}

p-ISSN: 2308-4944 (print) $\quad$ e-ISSN: 2409-0085 (online)

Year: $2015 \quad$ Issue: 11 Volume: 31

Published: $30.11 .2015 \quad \underline{\text { http://T-Science.org }}$

SECTION 20. Medicine.
Said Abdullaevich Salekhov

Professor, doctor of medical sciences Novgorod State University of Yaroslav Mudry,

Russia

ssalehov@mail.ru

Vladimir Igorevich Esaulov

Assistant, Department of Psychotherapy, Pirogov Russian National Research Medical

University, Russia v-esaulov@yandex.ru

Svetlana Olegovna Yablochkina

Candidate of medical sciences, Assistant professor,

Novgorod State University of Yaroslav Mudry,

Russia

iablochkina72@mail.ru

Inna Alexandrovna Korabelnikova graduate student,

Novgorod State University of Yaroslav Mudry,

Russia

inna.korabelnikova@gmail.com

\title{
INFLUENCE OF THE FIRST YEAR OF LIFE STRESS ON FORMATION OF EATING EHAVIOUR
}

\begin{abstract}
In this article the analysis of influence of psychological stress in first year babies on formation of eating behaviour and its reinforcement for implementation further in life. The analysis was based on fundamental provisions of psychophysiology, the theory of dominant, the theory of functional systems, regularities of development of the common adaptation syndrome, peculiarities of power supply in intensive impact of stress factors on the nervous system. Integrative approach in consideration of psychoemotional stress of the nursing mother, reaction of her organism to influence of a stress factor, transmittance of information about the stress to the baby with breast milk and immediate influence of decrease in lactation and introduction of supplemental feeding for the baby allowed to reveal two variants of the formed eating behaviour. On one hand, negative emotions of the nursing mother with transfer of humoral stress factors with breast milk, defining a psychoemotional state of the baby, combined with decrease in lactation contribute to formation of a "stress-hunger" link, as a result of which the "comfort eating" pattern is imprinted. On the other hand, decrease in lactation in the mother and introduction of supplemental feeding for the baby leads to the fact that against the discrepancy of adaptive resources of the baby's gastrointestinal tract to the change in character and structure of food the stress develops in the baby, which against influence of a stress factor and hunger - caused by impossibility to satisfy the increasing metabolic cost at the expense of increase in feeding, will form a survival strategy in the conditions of deficiency of recovery of energy and plastic resources from the outside. That is, prerequisites are created for formation of the eating behavior in stress providing decrease of the activity of energy-intensive processes of an organism including digestion and switch of metabolism to an energy-saving mode. Thus, the stress within the first year of life creates prerequisites for formation of eating behavior which can be implemented in the future, especially in stressful situations.

Key words: psychological stress, breastfeeding, introduction of supplementary feeding, stress in the nursing mother, stress in the baby, stress-hunger, comfort eating, self-eating

Language: Russian

Citation: Salekhov SA, Esaulov VI, Yablochkina SO, Korabelnikova IA (2015) INFLUENCE OF THE FIRST YEAR OF LIFE STRESS ON FORMATION OF EATING EHAVIOUR. ISJ Theoretical \& Applied Science 11 (31): $155-160$.

Soi: http://s-0-i.org/1.1/TAS-11-31-25 Doi: crossef http://dx.doi.org/10.15863/TAS.2015.11.31.25

\section{ВЛИЯНИЕ СТРЕССА 1 ГОДА ЖИЗНИ НА ФОРМИРОВАНИЕ ПИЩЕВОГО ПОВЕДЕНИЯ}

Аннотация: В статье, опираясь на фундаментальные положения психофизиологии, учения о доминанте, теории функциональных систем, закономерности развития общего адаптационного синдрома,
\end{abstract}


особенности энергетического обеспечения при интенсивном воздействии стрессоров на нервную систему был проведен анализ влияния психологического стресса у ребенка в течение первого года жизни на формирование пищевого поведения, его закрепления для реализации в последующей жизни. Интегративный подход в рассмотрении психоэмоционального стресса у кормящей матери, реакции ее организма на воздействие стрессора, передача информаџии о стрессе ребенку с материнским молоком и непосредственное влияние снижения лактации и введения прикорма у самого ребенка, позволил выявить два варианта формирующегося пищевого поведения. С одной стороны, негативные эмоции у кормящей матери с передачей гуморальных факторов стресса с материнским молоком, определяющции психоэмочииональное состояние, ребенка, в сочетании со снижением лактации способствуют формирование связки «стресс-голод», следствием чего закрепляется паттерн «заедание стресса». C другой стороны, при снижении лактации у матери и введении прикорма, при его несоответствии адаптивных ресурсов пищеварительной системы ребенка к изменению характера и структуры пищи стресс развивается у самого ребенка, что на фоне воздействия стрессора и голода, обусловленного невозможность восполнить возрастающие энергетические затраты за счет увеличения питания, будут формировать стратегию выживания в условиях дефицита восполнения энергетических и пластических ресурсов извне. То есть, создаются предпосылки для формирования пищевого поведения при стрессе, предусматривающего снижение активности энергозатратных процессов организма, в том числе и пищеварения, и переход метаболизма в энергосберегающий режим. Таким образом, стресс в течение первого года жизни создает предпосылки для формирования пищевого поведения, которое может реализоваться в будущем, особенно в стрессовых ситуациях.

Ключевые слова: психологический стресс, грудное вскармливание, введение прикорма, стресс у кормящей матери, стресс у ребенка, стресс-голод, заедание стресса, самопоедание.

Неуклонный рост частоты выявления ожирения в течение последних 30-40 лет во всем мире $[1,2,3,4,5,6,7,8]$ и явное омоложение данной патологии $[4,5,9]$ позволило отнести ее к «болезням образа жизни» или «болезням цивилизации» [10, 11]. Влияние данной патологии на все сферы жизни неуклонно растет. Это сделало ожирение и осложнения метаболического синдрома одной из приоритетных проблем теоретической и практической медицины, социологии, демографии, психологии и экономики.

Следует отметить, что на фоне избыточного веса наметилась отчетливая тенденция к увеличению риска летальности в молодом и среднем возрасте как у мужчин, так и у женщин $[12,13]$.

При этом лишь в 5-7\% случаев удается выявить этиологию ожирения, а остальные 9395\% относят к алиментарному (экзогенноконституциональному) ожирению невыясненной этиологии [10], что по сути своей парадоксально, учитывая затраты на многочисленные исследования, посвященные данной проблеме.

Вероятно, подходы к изучению данной проблемы, основанные на огромном объеме статистических данных, констатации мельчайших деталей результатов фундаментальных и клинических исследований без истинно междисциплинарного подхода могут привести к взаимной подмене этиологических и предрасполагающих факторов, интерпретации их значимости с позиции той или иной узкой научной специальности. Поэтому, до настоящего времени число теорий развития ожирения растет параллельно с ростом данной патологии [11].
При проведении анализа динамики факторов окружающей среды, негативно влияющих на человека, видно, что наиболее выраженный рост интенсивности негативного воздействия связан с психоэмоциональной напряженностью и информационным потоком, являющихся компонентами психологического стресса [11].

После первой публикации H. Selye в 1936 году [14,15] термин «стресс» прочно вошел в нашу жизнь. При этом рассмотрение психологического стресса, как разновидности общего адаптационного синдрома, часто проводят опираясь только на значимость психологических аспектов проблемы, без анализа энергетического обеспечения удовлетворения реакции на воздействие стрессора. Таким образом, при всей значимости психологических факторов, их рассматривают как нечто отдельное от жизнедеятельности организма в целом.

В связи с этим особого внимания заслуживает влияние факторов внешней среды и, в первую очередь психологического стресса, на формирование пищевого поведения в период внутриутробного развития и в раннем детском возрасте. При этом смена среды обитания и развитие в течение первого года жизни, когда происходит адаптация к существованию в новых условиях, могут играть определяющую роль в формировании копинг-стратегий, проявляющихся в том числе и в пищевом поведении, отражающем особенности энергетического обеспечения стресса в дальнейшей жизни.

Материалы и методы исследования:

Обоснование особенностей влияния психоэмоционального и информационного 
факторов, а по сути, психологического стресса на формирование пищевого поведения проводилось с опорой на учение о доминанте [16], основные положения теории функциональных систем [17] и развития общего адаптационного синдрома, физиологические изменения энергетического обеспечения мозга при интенсивной нагрузке [11], пирамиды потребностей А. Маслоу [18]. При этом рассмотрено воздействие психоэмоционального стресса на формирование пищевого поведения начиная с момента зачатия во время беременности и в течение первого года жизни.

Согласно учению о доминанте А.А. Ухтомского, при возникновении очага приоритетного возбуждения в центральной нервной системе основные ресурсы направляются на удовлетворение потребностей и запросов этого очага возбуждения из наиболее интенсивно функционирующего отдела мозга [16]. При этом запросы остальных отделов мозга обеспечиваются по остаточному принципу.

Согласно теории функциональных систем П.К. Анохина, при воздействии на организм формируется функциональная система, обеспечивающая адекватный ответ-реакцию на предъявляемые требования-запрос к организму, для достижения конечного положительного результата[17]. Структура и последовательность деятельности функциональной системы заключается в том, что:

-при поступлении входящей информации аппарат контроля формирует модель полезного результата;

-аппарат управления, объединяющий нервные центры и эндокринные органы определяет необходимые ресурсы для достижения полезного результата;

-исполнительный аппарат, представленный органами-аффекторами используя выделенные ресурсы, обеспечивает реализацию получения результата;

-аппарат контроля, по принципу обратной связи тестирует полученный результат на соответствие модели полезного результата.

Если соответствие достигнуто функциональная система завершает свою работу, а если нет, функциональная система вырабатывает новую стратегию достижения модели полезного результата с привлечением новых ресурсов организма. Цикл достижения модели полезного результата и обратной связи повторяется снова до получения соответствия полученного результата модели конечного положительного результата, запрограммированного центром контроля.

При многократно повторяющихся циклах достижения полученного результата запрограммированной модели и отсутствии соответствия с искомым, привлекаются все новые ресурсы и активизируются компенсаторноприспособительные механизмы организма, что в итоге сказывается на его функциональном состоянии, может привести к истощению, декомпенсации и развитию патологических состояний.

В основу теории о стрессе были положены наблюдения H. Selye, установившего, что на любое интенсивное воздействие организм реагирует одинаково, в виде развития общего адаптационного синдрома [15].

В пирамиде потребностей Абрахама Маслоу выделены ступени перехода от биологических потребностей к высшим [18]:

1 ступень - физиологические потребности, где стрессорами являются голод, жажда, бессоница, умственная и физическая усталость, чрезмерно быстрый темп жизни;

2 ступень - потребности безопасности и стабильности, где к стрессорам относятся страх и тревога потерять работу, провала на экзамене, страх за жизнь близких, страх смерти и т.д.;

3 ступень - потребность в принадлежности и любви, где стрессорами являются моральное и физическое одиночество, потеря близких людей или болезнь, неразделенная любовь;

4 ступень - потребность в уважении, где стрессорами будут крушение карьеры, провал на экзамене, невозможность реализовать свои амбиции, потеря уважения в обществе;

5 ступень - потребность в самореализации, где причинами стресса будут невозможность реализовать свои способности, вмешательство со стороны родителей или факторов внешней среды, которые препятствуют занятиям любимым делом, следовать своему признанию [19].

Следует отметить, что развитие стрессогенной ситуаци, обусловленной психоэмоциональным и информационным факторами, в зависимости от запроса, предъявляемого к организму, будет занимать одну или несколько ступеней в пирамиде потребностей А. Маслоу. При этом переход на более приоритетные ступени, обеспечивающие базовые потребности (обеспечение жизнедеятельности организма - I ступень и потребность в безопасности и стабильности - II ступень) могут являться последствиями реакции организма на первичное воздействие [20].

На фоне интенсивной деятельности ЦНС отмечается увеличение потребления мозгом кислорода и глюкозы, что обеспечивает удовлетворение возрастающего запроса в энергии. При этом недостаток в поступлении либо кислорода, либо глюкозы, обеспечивающий аэробный катаболизм глюкозы в нервных клетках сопровождается энергетическим дефицитом, следствием чего будет торможение [11]. 
Для понимания особенностей формирования пищевого поведения следует учитывать, что развитие организма и научение его реагированию на внешние воздействия происходит по периодам.

Наименее изучен период от зачатия и до рождения [21]. Далее следует период от рождения до 1 года, которому и посвящено это исследование.

Результаты исследования:

От рождения до 1 года основными потребностями ребенка являются потребности в питании, тепле, принятии, заботе, защите, направленными на обеспечение жизнедеятельности и безопасности (I и II ступень пирамиды потребностей А. Маслоу).

На первом году жизни ребенка при его плаче основным стереотипом поведения матери, доведенным до уровня рефлекса, является кормление грудью или бутылочкой с молоком, которое часто носит седативный успокаивающий эффект.
В дикой природе котенок или щенок начинают скулить, когда им холодно, они голодны, либо испытывают какой-то дискомфорт. При этом дискомфорт может быть обусловлен, помимо голода, другими причинами.

По аналогии с животными, ребенок может заплакать либо когда он голоден, либо когда он испытывает дискомфорт вследствие иных причин.

Плач ребенка не всегда связан только с чувством голода. Его причиной может быть любое другое дискомфортное состояние. Насильственное кормление ребенка в это время, при отсутствии чувства голода, формирует связку «дискомфорт-кормление», что может создать условия для закрепления рефлекса «дискомфортпитание», являющегося одним из факторов формирования пищевого поведения.

На рисунке 1 представлена схема влияния стресса первого года жизни на формирование пищевого поведения.

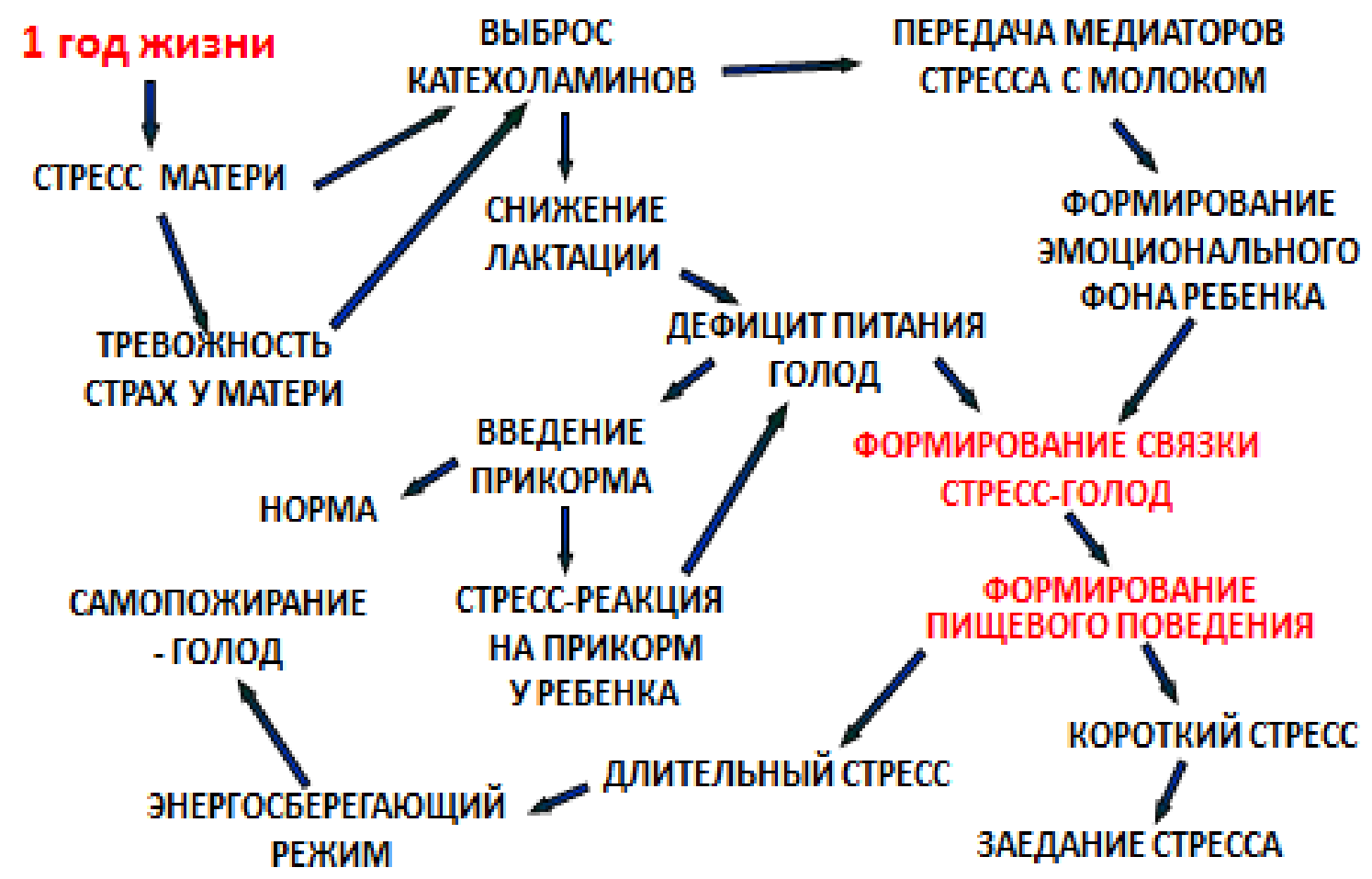

Рисунок 1 - Влияние стресса 1 года жизни на формирование пищевого поведения.

Как видно из рисунка, большое значение будет иметь психоэмоциональное состояние матери, особенно наличие негативных стрессовых ситуаций в период грудного вскармливания. Так, психоэмоциональное напряжение и, связанный с ним стресс матери будет сопровождаться выбросом катехоламинов в кровь, что происходит при типичной реакции на воздействие стрессора. При грудном вскармливании катехоламины и другие медиаторы стресса будут поступать к ребенку с материнским молоком, что будет формировать его эмоциональное состояние. 
В то же время, на фоне стрессовой ситуации у матери может снизиться лактация, что, в сочетании с поступлением гуморальных факторов стресса в материнском молоке к ребенку, а при его недостаточном количестве, закрепляет у него паттерн «стресс-голод-потребность заедания».

Когда же при дискомфортных и стрессовых ситуациях имеет место поступление дополнительного или повышенного питания для удовлетворения возросших энергетических запросов, формируется концепция заедания любого стресса и любого дискомфорта непосредственно во время воздействия стрессора.

Не менее важным фактором, влияющим на формирование пищевого поведения, будет введение прикорма на фоне снижения лактации. При этом реакция ребенка на введение прикорма может быть двоякой. С одной стороны усвоение прикорма может происходить без дискомфортных явлений и расстройства пищеварения, что можно отнести к благоприятному сценарию.

Однако, вследствие изменения структуры пищи и ее состава в сочетании с недостаточно адаптированной пищеварительной системой к подобным изменениям, введение прикорма может стать стрессом уже для самого ребенка. Более того, на фоне стандартной реакции на стресс со стороны организма, расстройство пищеварения будет сопровождаться сохранением, а возможно и прогрессированием дефицита питательных веществ, что в еще большей степени будет закреплять связку «стресс-голод».

Соответственно, в зависимости от продолжительности и постоянства стрессовой ситуации могут быть сформированы два сценария пищевого поведения.

При коротких эпизодах стресса, чередующихся с ремиссиями, будет закрепляться связка «стресс-голод-заедание стресса». При этом заедание будет происходить не при нарастании стресса, а на фоне его регрессии и в периоды ремиссий.

В тех случаях, когда стресс протекает непрерывно длительный период времени, организм будет адаптироваться к резкому снижению поступления питательных веществ за счет исключения наиболее энергозатратных процессов, одним из которых является пищеварение. Таким образом во время стресса может включаться процесс «самопоедания». При этом на фоне длительного воздействия стрессора, в сочетании с нарушением пищеварения, доминантным становится выживание в условиях дефицита питания.

При этом в соответствии с теорией функциональных систем может выработаться стратегия, позволяющая использовать имеющиеся в распоряжении организма внутренние пластические и энергетические ресурсы.
Когда будет отмечаться регрессия интенсивности стресса, возможно увеличение потребления пищи с формированием запаса внутренних энергетических ресурсов в виде накопления жировой ткани, с учетом вероятного повторения стрессовой ситуации. При этом на фоне дискомфорта и стресса, параллельно, формируется чувство голода, стимулирующее пищедобывательное поведение, обусловленное либо недостатком пищи, либо увеличением потребности в энергетических ресурсах для адекватного удовлетворения запроса, предъявляемого организму дискомфортной или стрессовой ситуацией.

Альтернативно этому при часто повторяющихся длительных стрессовых ситуациях может наблюдаться стойкий переход метаболизма в энергосберегающий режим, следствием чего будет дефицит массы тела и явления саркопении. То есть, когда имеет место дефицит пищи, для восполнения энергетических затрат, необходимых для удовлетворения запроса внешней среды, происходит мобилизация внутренних энергетических ресурсов. Это создает предпосылки для формирования внутренней концепции «самопоедания» при запросе внешней среды к организму.

Часто повторяющиеся и продолжительные периоды воздействия стрессоров (по сути хронический стресс), будут причиной долговременной перестройки метаболизма организма с переходом в энергосберегающий режим. Периоды ремиссии могут восприниматься уже как некое отклонение от нормы, с сохранением стратегии энергосберегающего режима метаболизма.

Таким образом, при влиянии стресса 1 года жизни на формирование пищевого поведения можно отметить формирование двух параллельных связок «стресс-голод-питание» (заедание) и «стресс-голод-энергосберегающий режим-самопоедание-отсроченное заедание стресса», либо «стресс-голод-энергосберегающий режим-самопоедание-истощение и закрепление энергосберегающего режима».

В отличие от этого, бережное щадящее отношение близких к маме и ребенку, создание для них комфортных условий в этот сложный для адаптации период, будет способствовать формированию нормального режима питания и, соответственно, пищевого поведения.

Учет механизмов формирования пищевого поведения при воздействии стресса на ранних этапах развития может быть полезен в понимании возникновения патологического пищевого поведения, и его профилактике. Для формирования здорового пищевого поведения целесообразно воспитание и профилактическое 
обучение будущих родителей, направленное на понимание ими правильного построения взаимоотношений, а также бережного отношения к женщине как во время беременности, так и после рождения ребенка.

\section{References:}

1. Friedman JM (2000) Obesity in the new millennium / J.M. Friedman // Nature, 2000. 404. pp. 632-634.

2. Gómez-Ambrosi J (2012) Clinical usefulness of a new equation for estimating body fat / J. Gómez-Ambrosi, C. Silva, V.Catalán et al. // Diabetes Care, 2012. Vol. 35. pp. 383-388.

3. Heindel JJ (2013) The Perfect Storm for Obesity / J.J. Heindel, T.T. Schug // Obesity, 2013. Vol. 21, Issue 6. pp. 1079-1080.

4. Kim JE (2013) Childhood obesity as a risk factor for bone fracture: A mechanistic study / J.-E. Kim, M.-H. Hsieh, B.K. Soni et al. // Obesity, 2013, Vol. 21, Issue 7. pp. 1459-1466.

5. Lin H (2013) Food reinforcement partially mediates the effect of socioeconomic status on body mass index / H. Lin, K.A. Carr, K.D. Fletcher, L.H. Epstein // Obesity, 2013. Vol. 21, Issue 7. pp. 1307-1312.

6. Rosas LG (2012) Practical research strategies for reducing social and racial/ethnic disparities in obesity / L.G. Rosas, R.S. Stafford // Int. J. Obes. Suppl., 2012. N 2. pp. 16-22.

7. Wang Y (2007) The obesity epidemic in the United States-gender, age, socioeconomic, racial/ethnic, and geographic characteristics: a systematic review and meta-regression analysis / Y.Wang, M.A. Beydoun // Epidemiol Rev., 2007. Vol. 29. pp. 6-28.

8. Yoshimoto S (2013) Obesity-induced gut microbial metabolite promotes liver cancer through senescence secretome / S. Yoshimoto, T.M. Loo, K. Atarashi et al. // Nature, 2013. 499. pp. 97-101.

9. Flores G (2013) Factors predicting severe childhood obesity in kindergarteners / G. Flores, H. Lin // Int. J. Obes., 2013. Vol. 37. pp. 31-39.

10. Veber VR (2011) Ozhirenie (etiologiya, patogenez, klinicheskie rekomendacii / V.R. Veber, M.N. Kopina. Velikiy Novgorod, 2011. $203 \mathrm{p}$.

11. Salehov SA (2014) Psihoemocionalnaya informacionno-energeticheskaya teoriya ozhireniya: - Velikiy Novgorod-Almaty, 2014. $-180 \mathrm{p}$.

12. Bray GA (2013) Why do we need drugs to treat the patient with obesity? / G.A. Bray // Obesity, 2013. Vol. 21, Issue 5. pp. 893-899.

13. Mehta T (2013) Does obesity associate with mortality among hispanic persons? Results from the national health interview survey / $\mathrm{T}$. Mehta, R. Mc Cubrey, N.M. Pajewski et al. // Obesity, 2013.Vol. 21, Issue 7. pp. 1474-1477.

14. Selye H (1936) A Syndrome produced by Diverse Nocuous Agents. // Nature 138, 32 (4 July 1936) | doi:10.1038/138032a0.

15. Selye H (1938) Adaptation Energy // Nature 141, $926 \quad(21 \quad$ May 1938) doi:10.1038/141926a0.

16. Uhtomskiy AA (1923) Dominanta kak rabochiy princip nervnyh centrov / A.A. Uhtomskiy // Russk. fiziol. zhurn., T. VI, vyp. 1-3, 1923, pp. 31-45.

17. Anohin PK (1970) Teoriya funkcionalnoy sistemy / P.K. Anohin // Uspehi fiziol. nauk. 1970. T. 1, № 1. pp. 19-54.

18. Maslou AG (1999) Dalnie predely chelovecheskoy psihiki / Perev. s angl. A. M. Tatlybaevoy. - SPb.: Evraziya, 1999. -432p.

19. Scherbatyh YV (2012) Psihologiya stressa i metody korrekcii. 2-e izd. - SPb.: Piter, 2012. $256 \mathrm{p}$.

20. Salehov SA, Gordeev MN, Bizina TV, Maksimyuk NN (2015) Ierarhiya psihoemocionalnogo i informacionnogo faktorov v strukture ekzamenacionnogo stressa // Sovremennye problemy nauki i obrazovaniya. - 2015. - № 5; Available: http://www.scienceeducation.ru/128-21959 10.11.2015).

21. Salekhov SA, Esaulov VI, Yablochkina SO (2015) Influence of expectant mothers negative stress on coping strategy and their energy supply forming // ISJ Theoretical \& Applied Science -2015.- №10(30): pp. 111-116. Soi: http://s-o-i.org/1.1/TAS-10-30-24 Doi: http://dx.doi.org/10.15863/TAS.2015.10.30.24 